\title{
Synthesis of New Fluorinated 1,2,4-Triazino $[3,4-b][1,3,4]$ thiadiazolones as Antiviral Probes-Part II-Reactivities of Fluorinated 3-Aminophenyl-1,2,4-triazinothiadiazolone
}

\author{
Mohammed Saleh Tawfek Makki, Reda Mohammady Abdel Rahman, Ola Ahmad Abu Ali* \\ Chemistry Department, Faculty of Science, King Abdul Aziz University, Jeddah, Kingdom of Saudi Arabia \\ Email: ${ }^{*}$ lolo aa4@hotmail.com
}

Received 23 June 2015; accepted 5 September 2015; published 8 September 2015

Copyright $@ 2015$ by authors and Scientific Research Publishing Inc.

This work is licensed under the Creative Commons Attribution International License (CC BY). http://creativecommons.org/licenses/by/4.0/

\section{(c) (i) Open Access}

\begin{abstract}
Some new fluorinated 3-N-acyl/3-N-alkylaminophenyl-1,2,4-triazino[3,4-b][1,3,4]thiadiazolones (2-12) have been obtained from treatment of 2-(4'-fluorophenyl)-6-(2'-amino-5'-fluorophenyl)1,2,4-triazino[3,4-b][1,3,4] thiadiazol-4-one(1) with active functional oxygen, sulfur and halogen compounds in different conditions. Former structures of the products have been characterized from elemental and spectral data (UV, IR, NMR and Mass). The new products were evaluated as potential anthelmintic drugs.
\end{abstract}

\section{Keywords}

Synthesis, Fluorinated 1,2,4-Triazinothiadiazolones, Anthelmintic Drugs

\section{Introduction}

The treatment of infectious diseases still remains an important and challenging problem because of a combination of many factors including emerging infectious diseases and the increasing number of multi-drug resistant microbial pathogens [1]-[4]. There is real perceived need for the discovery of new compounds endowed with biocidal activity. Through the various molecules designed and synthesized for this aim, it was demonstrated that fluorinated 1,2,4-triazine fused with 1,3,4-thiadiazole systems. The introduction of fluorine atom to the heterocyclic systems improves or enhances the medicinal properties [5]-[9]. On the other hand, most of heterocyclic nitrogen systems bearing an amino-groups exhibit a wide spectrum of biological activities [10]. And their use is

\footnotetext{
${ }^{*}$ Corresponding author.

How to cite this paper: Makki, M.S.T., Rahman, R.M.A. and Ali, O.A.A. (2015) Synthesis of New Fluorinated 1,2,4-Triazino [3,4-b][1,3,4]thiadiazolones as Antiviral Probes-Part II-Reactivities of Fluorinated 3-Aminophenyl-1,2,4-triazinothiadiazolone. International Journal of Organic Chemistry, 5, 153-165. http://dx.doi.org/10.4236/ijoc.2015.53017
} 
as starting material.

Recently, synthesis and chemistry of 1,3,4-thiadiazoles as biocidal agents have been reviewed [11] [12]. Also, 1,2,4-triazine derivatives have been synthesized and evaluated as biological and pharmacological probes [13]-[15].

Abdel-Rahman et al. [16], reported that 1,2,4-triazino[3,4-b][1,3,4]thiadiazolones (Figure 1) used as anti HIV and anticancer drugs. In contamination of our work in these researches for new biocidal agents [17], the present investigation reports the preparation of fluorinated 3-substitutedamino-1,2,4-triazino[3,4-b][1,3,4]thiadiazolones starting from the corresponding 3 -amino analogus, as potential anthelmintic drugs.

\section{Results and Discussion}

3-Substituted-1,2,4-triazines and their azole-fused analogs reacts with bifunctional compounds give a more stable polycyclic systems depends on the triazine substrate nature. Search for new bioactive compounds, the main aim of the present work is preparation of fluorinated 3-substituted amino-1,2,4-triazino[3,4-b][1,3,4]thiadiazolones in view of their pharmacological properties.

Thus, addition of cyclohexyl isocyanate and/or 4-fluorophenyl-isothiocyanate to 3-(2'-amino-5'-fluoro-phenyl) -7-(4'-fluorophenyl)-1,2,4-triazino[3,4-b][1,3,4]thiadiazol-4-one (1) [17], (Scheme 1) in warm DMF afforded N(cyclohexyl)-N'-(4'-oxo-1,2,4-triazino[3,4-b][1,3,4] thiadiazol-7'-(4'-fluorophenyl)-3-(4'-fluorophenyl) urea (2) and/or N-(4'-fluorophenyl)-N'-(4'-oxo-1',2',4'-triazino[3,4-b][1,3,4]thiadiazole-7'-(4"-fluoro-phenyl)-3'-(4"-fluorophenyl)thiourea (3) (Scheme 2). Formation of compound 3 may be tack's place via a nucleophilic attack of $\mathrm{NH}_{2}$ group to a more electrophilic carbon of isothiocyanate.

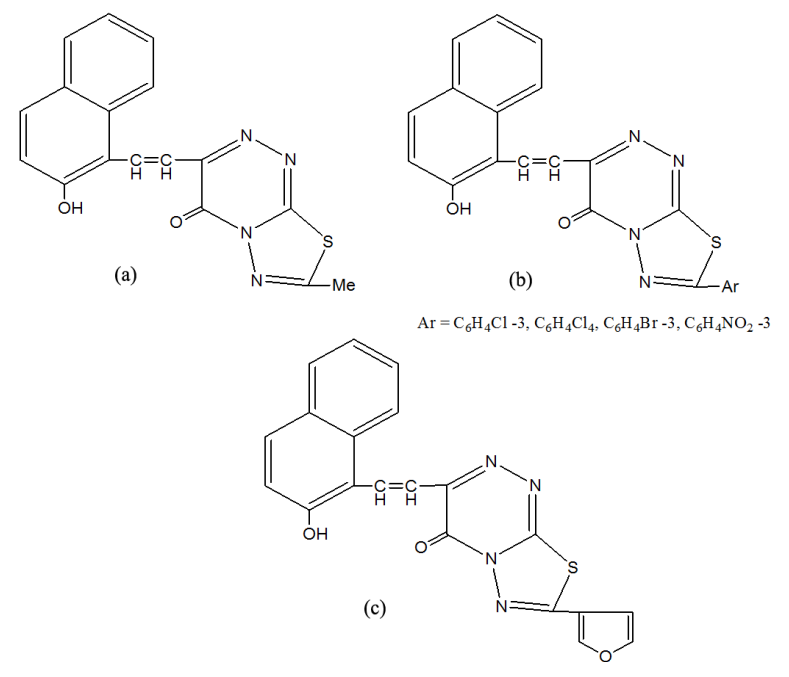

Figure 1. Some 1,2,4-triazino[3,4-b][1,3,4]thiadiazolones as anti HIV and anti-cancer drugs.

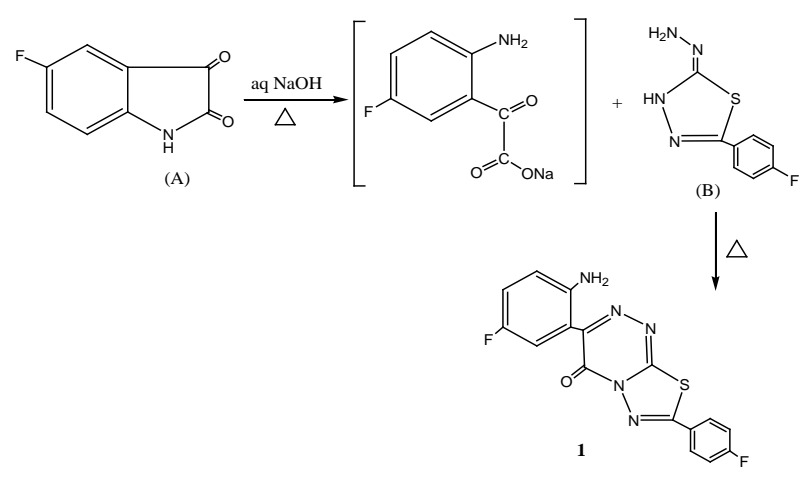

Scheme 1. Synthesis of compound $\mathbf{1}$. 
Acylation of compound 1 using acetyl chloride (DMF) [18] and /orethyltrifluoroacetate in reflux (THF) [19] yielded 3-[(2'-acetylamino)-5'-fluorophenyl]-7-(4'-fluorophenyl)-1,2,4-triazino[3,4-b][1,3,4]thiadiazol-4-one (4) and/or 3-(2'-trifluoroacetylamino-5'-fluoro-phenyl-7-(4'-fluorophenyl)-1,2,4-triazino[3,4-b][1,3,4]thiadiazol-4-one (5) (Scheme 3). Also, self cyclo-condensation of compound 1 via boiling with DMF furnished thiadiazolo-1,2,4triazinoindole derivative 6 (Scheme 3).

Bonded phosphorus atoms with $\mathrm{S}, \mathrm{O}, \mathrm{N}$ and $\mathrm{C}$-atoms of heterocyclic system enhance their biocidal properties as herbicides, pesticides, insecticides and molluscicidal agents [20]-[22]. With this observations, the present work aims to synthesize of new fused heterobicyclicbearing fluorine and phosphorus atoms through phosphorylation of compound $\mathbf{1}$ with diphenyl phosphoryl chloride in warm DMF to give 3-(2'-diphenylphosphatoamino-

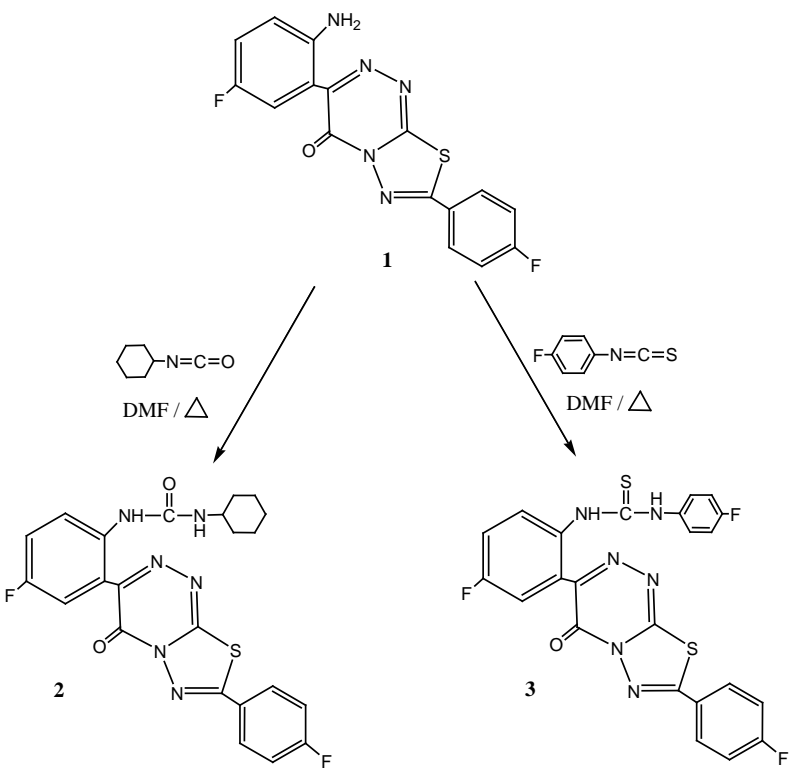

Scheme 2. Synthesis of compounds 2 and 3.

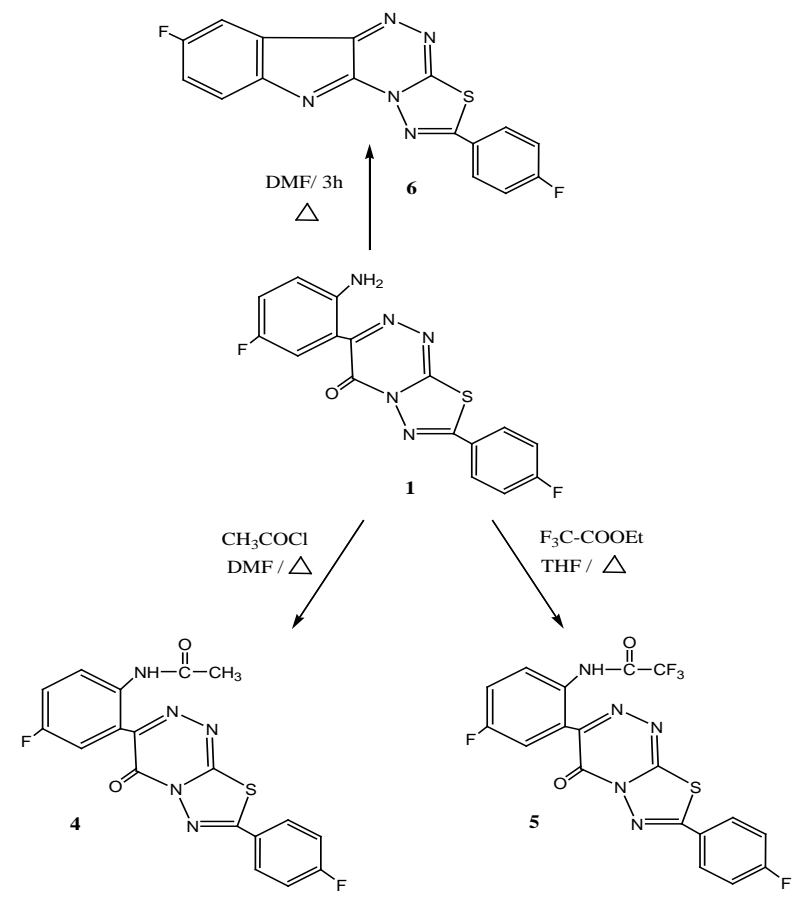

Scheme 3. Synthesis of compounds 4-6. 
5'-flu-orophenyl)-7-(4'-fluorophenyl)-1,2,4-triazino[3,4-b][1,3,4]thiadiazol-4-one (7) (Scheme 4). Due to a highly with drown of $\mathrm{P}$ of phosphate moiety, the chlorine atom is very labile. Thus, simple $\mathrm{Nu}^{-}$attack of $\mathrm{NH}_{2}$ to $\mathrm{P}$ atom afforded the aminophosphate derivative.

Full fluorinated 3-[5'-fluoro-2'-(4"-fluorobenzoylamino)phenyl]-7-(4'-fluorophenyl)-1,2,4-triazino[3,4-b][1,3,4] thiadiazole-4-one (8) was obtained from treatment of compound 1 with 4-fluorobenzoylchloride in warm DMF (Scheme 4).

Due to a higher nucleophilicity of amino-group and the better displacement of labile chlorine atom of halo acids the interested point in this investigation is a simple nucleophilic attack of amino-group of compound $\mathbf{1}$ to a higher electrophilic carbons of $\alpha$-haloacids as monochloroacetic acid and/or 1,1-dichloroacetic acid in warm DMF, yielded [23] 3-(5"-fluoro-2'-carboxymethylanilino)-7-(4'-fluorophenyl)-1,2,4,-triazino[3,4-b][1,3,4]thia-diazol-4one (9) and/or 3-(5'-fluoro-2'-carboxymithinicanilino)-7-(4'-fluorophenyl)-1,2,4-triazino[3,4-b][1,3,4] thiadiazol4-one (10) (Scheme 5).

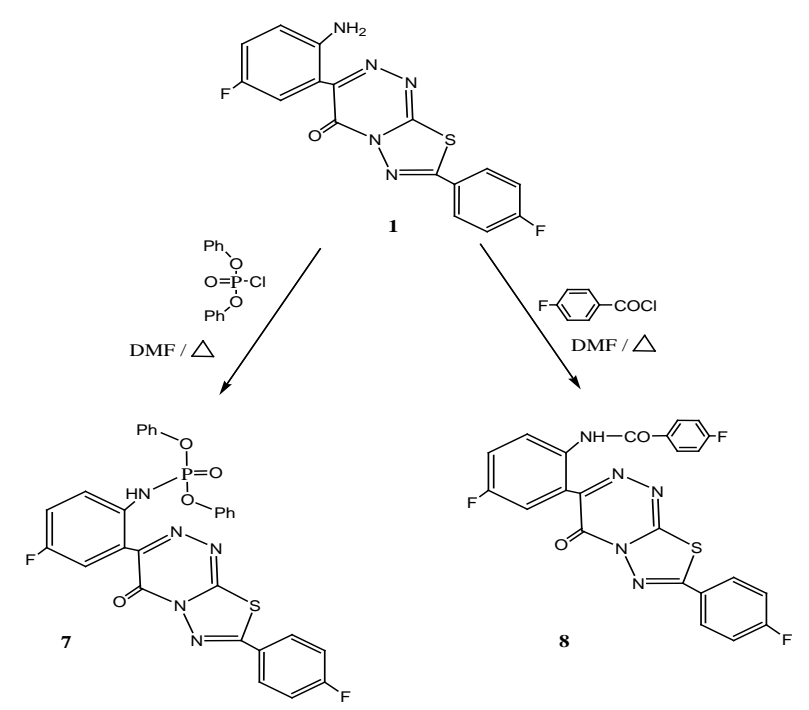

Scheme 4. Synthesis of compounds 7 and $\mathbf{8}$.
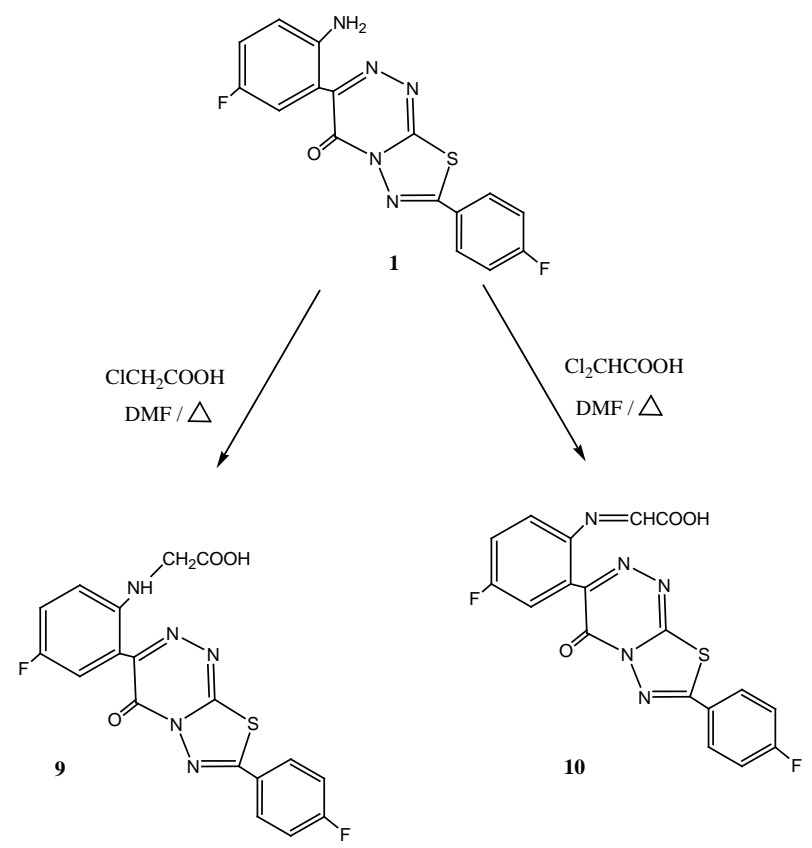

Scheme 5. Synthesis of compounds $\mathbf{9}$ and $\mathbf{1 0}$. 
Alkylation, reaction of 3-amino-1,2,4-triazinothiadiazolone 1with chloroacetonitrile in warm DMF produced [24] the 3-(5'-fluoro-2'-(cyanomethylanilido)-7-(4'-fluorophenyl)-1,2,4-triazino[3,4-b][1,3,4]thiadiazol-4-one (11) (Scheme 6). Acidic hydrolysis of $\mathbf{1 1}$ by reflux with dil. $\mathrm{HCl}$ afforded compound $\mathbf{9}$. Decarboxylation of $\mathbf{9}$ via warm with sodium bicarbonate solution, 3-(5'-fluoro-2'-(methylanilido)-7-(4'-fluorophenyl)-1,2,4-triazino[3,4-b] [1,3,4] thiadiazol-4-one (12) was isolated. The compound $\mathbf{1 2}$ was also, obtained from stirring of compound $\mathbf{1}$ with MeI in $1 \% \mathrm{KOH}$ solution (Scheme 6).

The adducts formed by reactions of nitrogen containing aromatic heterocycles with various electrophilic carbon, may be stable systems, or they can undergo further transformation, such as aromatization.

Abdel-Rahmanetal reported [1] [25], fluorine substituted thiobarbituric acid derivatives use as anti HIV-1 and cyclin dependent kinase 2 (CDK2) for cell tumor division, thus ring closure reaction of compound 3 with malonic acid in boiling with glacial acetic acid afforded the fluorine substituted N,N'-disubstitutedthiobarbituric acid 13 (Scheme 7). Formation of compound $\mathbf{1 3}$ was deduced from ring closure reaction of substituted thiourea 3 with malonic acid (Figure 2).

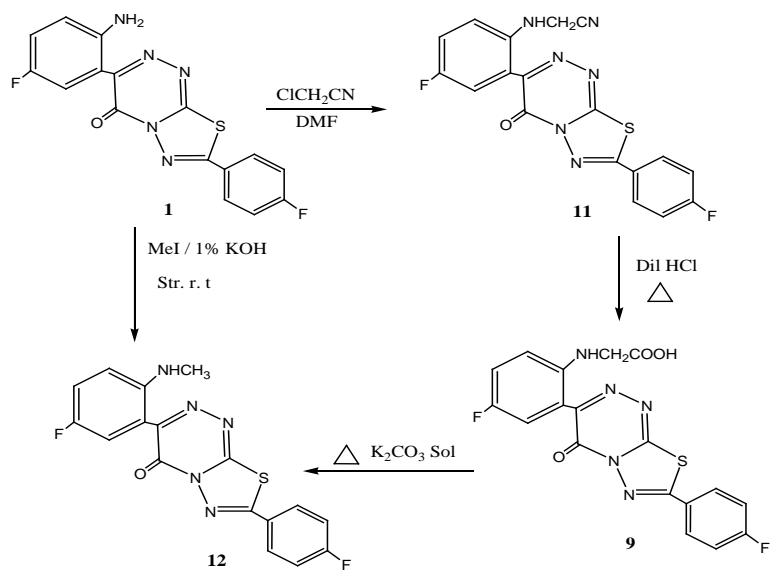

Scheme 6. Synthesis of compounds 11 and 12.<smiles>O=C(O)CC(=O)OCc1ccc(F)cc1-c1nnc2sc(-c3ccc(F)cc3)nn2c1=O</smiles>

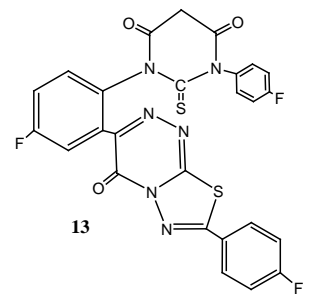

Scheme 7. Synthesis of compounds 13.<smiles>CC(C)N(C)C(=O)CC(=O)O</smiles>

Figure 2. Formation of compound 13 from 3. 
Former structures of the fluorinated 1,2,4-triazino[3,4-b][1,3,4]thiadiazolonederivatives have been established by help of their correct elemental analysis and spectral measurements:

1) UV absorption spectra of most N-acyl/phosphoryl derivatives for example 4, 6 and 7 recorded $\lambda_{\max } 304 \mathrm{~nm}$ as parent amino-derivatives $\mathbf{1}$, which is may be that electronic inhibition over $\mathrm{NH}_{2}$ by high electronic acceptor acyl, and/or phosphoryl. On the other hand, UV absorption spectra of compound $\mathbf{5}$ and $\mathbf{8}$ showed $\lambda_{\max }$ at 311 , and $375 \mathrm{~nm}$ respectively, which is may be the introduction of $\mathrm{COCF}_{3}$ and/or $\mathrm{COC}_{6} \mathrm{H}_{4} \mathrm{~F}-\mathrm{p}$ to an amino group of $\mathbf{1}$. Thus $\mathrm{NH}$ proton of these compounds is highly acidic character. In addition, UV absorption spectrum of $\mathbf{1 3}$ showed an additive $\lambda_{\max }$ at $410 \mathrm{~nm}$, which attribute to formation of fluorinated thiobarbituric acid bearing of 1,2,4-triazino-1,3,4-thiadiazinone moiety.

2) IR-spectra of all the obtained compounds (expected 6, 10, and13) recorded the absorption bands at 3200 $3100 \mathrm{~cm}$ for $\mathrm{NH}$ functional group, while, that of compounds showed an two $\mathrm{C}=\mathrm{O}$ of $\mathrm{NH}$ acyl and 1,2,4-triazinone at $1690-1650 \mathrm{~cm}^{-1}$. All the synthesized showed a charactic bands of stretching and bending of C-F at $\gamma 1250$ and $720 \mathrm{~cm}^{-1}$. Only the compounds 7, exhibited the presence of $\mathrm{P}=\mathrm{O}$ and C-O-Ar at 1097 and $1016 \mathrm{~cm}^{-1}$, while the compounds 4, 9, 11, and 12 showed the absorption bands of aliphatic groups at 2880 and $1440 \mathrm{~cm}^{-1}$. Some compounds as 5, 7, 8, and 11recorded a lack's of NH functional group, which is may be formation a type of H-bonding (Figure 3).

3) NMR spectra of the new synthesized compounds was confired that structures.

a) ${ }^{1} \mathrm{H}$ NMR spectra of all the obtained systems exhibited the presence of a resonated signals at $\delta 8.73 \mathrm{ppm}$ for $\mathrm{NH}$ proton (s), in addition, $\delta$ at $7.97-7.94$ and 7.38 - 7.35 ppm for 7 aromatic protons (m). On the other hand, compounds $2,4,9,11,12,13$ showed signals at $2.51 \mathrm{ppm}$ for an aliphatic protons $\left(\mathrm{COCH}_{3}, \mathrm{CH}_{2} \mathrm{CN}, \mathrm{CH}_{2}-\right.$ $\left.\mathrm{COOH}, \mathrm{CH}_{2} \mathrm{CO}\right)(\mathrm{J}=8.5)$. Only the compound 10 recorded $\delta$ at $8.8 \mathrm{ppm}$ for $\mathrm{N}=\mathrm{CH}$ proton (s).

4) ${ }^{13} \mathrm{C}$ NMR spectra of all the prepared compounds showed a resonated signals of fluorinated 1,2,4-triazino[3,4-b][1,3,4]thiadiazinone carbons at $\delta$ 164-163 (C=O), $135(\mathrm{C}-\mathrm{F}), 130-120$ (aromatic C), $116(\mathrm{C}=\mathrm{N})$ $\mathrm{ppm}$. Only the compound 3 showed a resonated signals at $180 \mathrm{ppm}$ attribute to $\mathrm{C}=\mathrm{S}$. On the other hand most
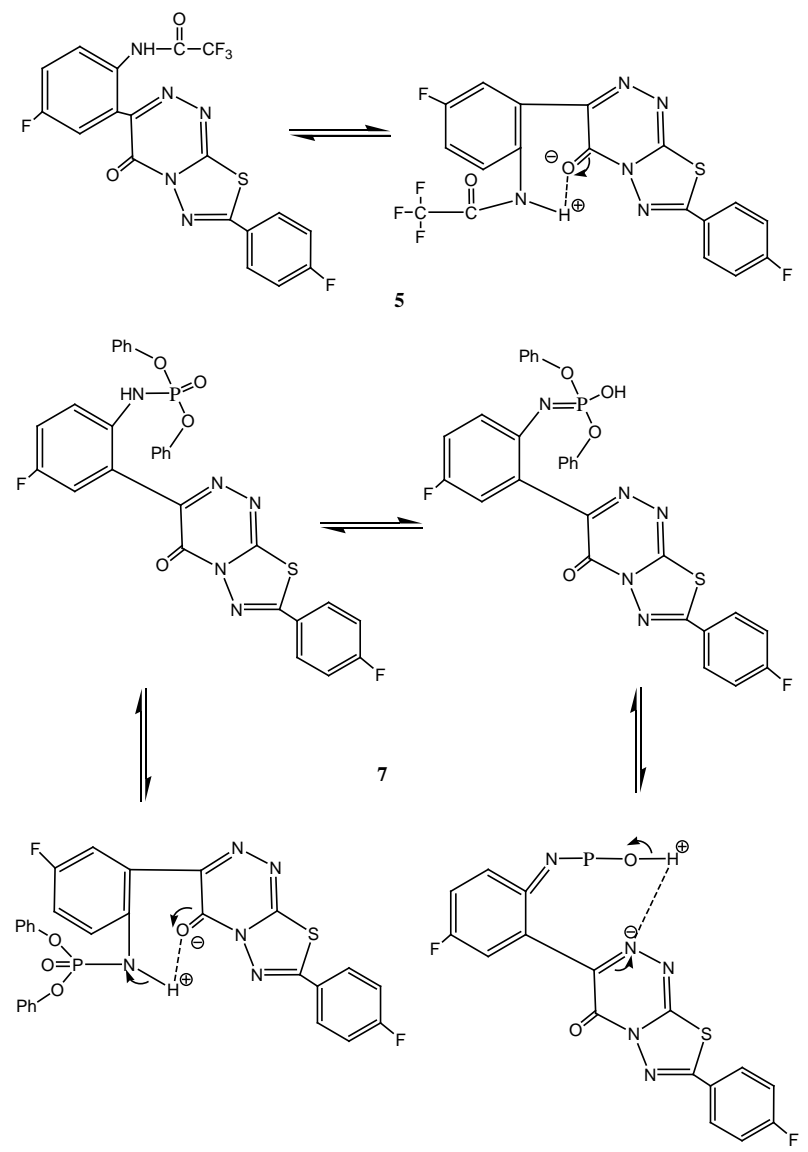

Figure 3. A possible structural formula of compounds 5, 7. 
of the new compounds showed an additional $\delta 160$ ppm for new NHCO carbons.

5) Mass fragmentation study of some new systems $\mathbf{5}$ showed the molecular ion peak, with a base peak at m/e 95 and $190(100 \%)$. The first base peak is 4-fluorophenylradical while the other is 4,4'-difluorobiphenyl radical.. Also present $\mathrm{M}^{+2}$ is attributed to $\mathrm{S}$ and $\mathrm{F}$ isotopic (Figure 4).

On the other hand, mass fragmentation study of compound 7 recorded a molecular ion peak at m/e 590 with a base peak at m/e 248 as $\mathrm{C}_{12} \mathrm{H}_{11} \mathrm{NPO}_{3}$ iminophosphato ion radical with 4-fluorophenyl cation at $95 \%$. Stability of a base peak is may be due to a higher stability of $\mathrm{N}=\mathrm{P}$ group, which supported by donation and back-donation between $\mathrm{N}$ and $\mathrm{P}$ atoms (Figure 5).

\section{Conclusion}

The present work describes a facile and simple nucleophilic attack of amino group bearing fluorine substituted 1,3,4-thiadiazolo[2,3-c][1,2,4]triazine(1) to active electrophilic reagents via addition, fluorinated acylation/ aroylation, phosphorylation and/or alkylation reactions. Substituted amino derivatives were obtained coupling with electronic modifications overall the molecule which led to potential anthelmintic activity. Among the new compounds $\mathbf{5}>\mathbf{7}>\mathbf{1}$ exhibit a higher activity (55\% - 60\%), while other $\mathbf{1 3}>\mathbf{9}>\mathbf{6}>\mathbf{4}(45 \%-48 \%)$ showed a moderate activity towards $\mathrm{N}$. brasiliensis virus. A higher activity of compounds $\mathbf{5}$ and $\mathbf{7}$ may be attributed to bourdation of $\mathrm{COCF}_{3}$ and/or phosphoryl groups with amino groups of start $\mathbf{1}$.

\section{Experimental}

Melting points of the products were determined on Stuart $\mathrm{SMP}_{3}$ (UK) and uncorrected. UV absorption spectra $\left(\lambda_{\max } \mathrm{nm}\right)$ were recorded in DMF on Shimadzu UV and visable 310 IPC-spectro-photometer. A Perkins Elmer Model RXI-FT IR system 55529 used for recording IR spectra of the prepared compounds $\gamma \mathrm{cm}^{-1}$. A Brucker advanced D P X $400 \mathrm{MHz}$ model using TMS as internal standard used for recording the ${ }^{1} \mathrm{H}$ and ${ }^{13} \mathrm{C}$ NMR spectra of the compounds on deuterated $\left(\mathrm{CDCl}_{3}, \mathrm{~d}_{6}, \delta \mathrm{ppm}\right)$. Mass spectrum was measured on GCMS Q 1000 Ex at $70 \mathrm{eV}$. Elemental microanalysis were performed by the microanalytical at Cairo-University, Egypt.

\subsection{3-(2'-Amino-5'-fluorophenyl)-7-(4'-fluorophenyl)-1,2,4-triazino[3,4-b][1,3,4] thiadi-azin-4-one (1)}

Equimolar amounts of 5-fluoroisatin (in 5\% aqueous $\mathrm{NaOH}$ ), and 2-hydrazino-2-(4'-fluorophenyl)-4H-1,3,4-

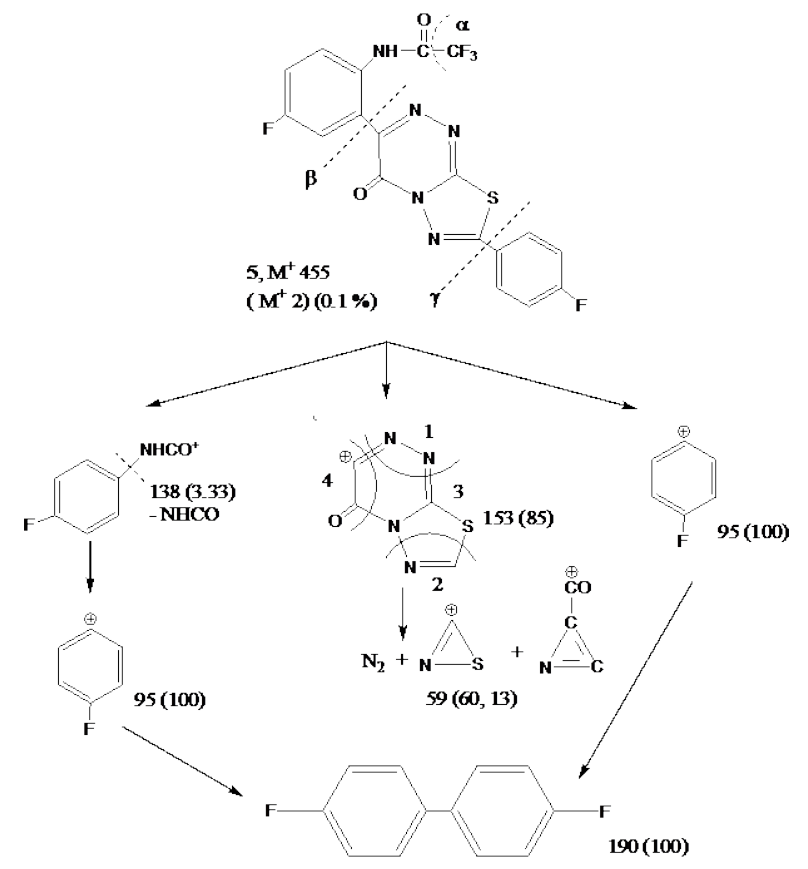

Figure 4. Mass fragmentation pattern of compound 5. 


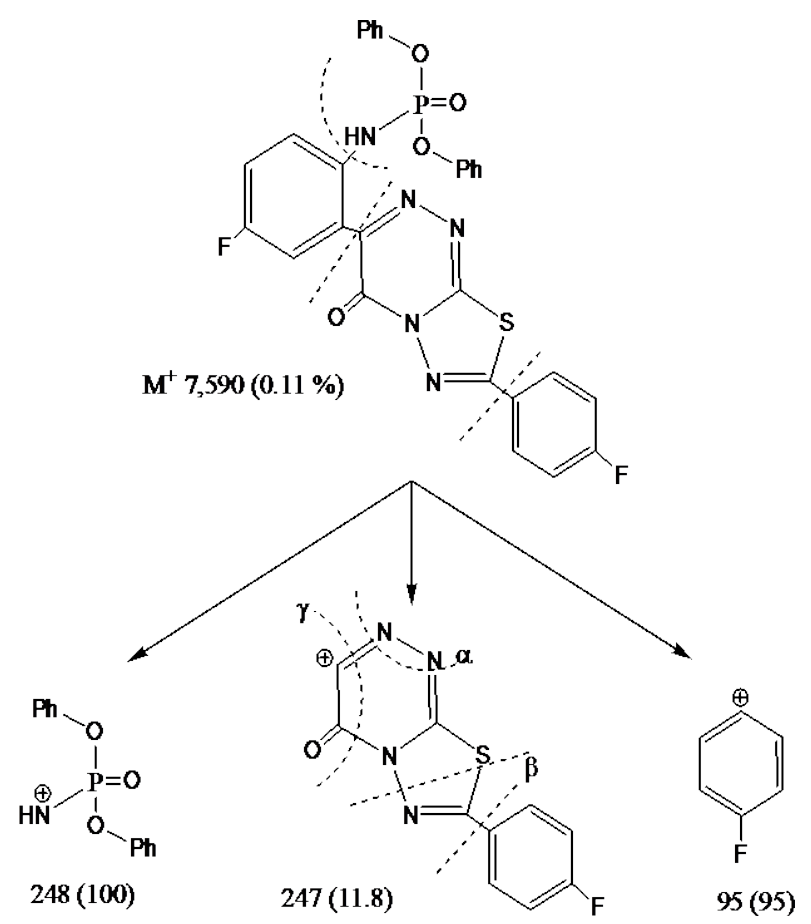

Figure 5. Mass fragmentation pattern of compound 7.

thiadiazole and reflux for $3 \mathrm{~h}$, cooled then poured on to ice- $\mathrm{HCl}$. The solid thus obtained filtered off and crystallized from dioxan to give 1 faint yellow crystals, yield $77 \%$, m.p. $189^{\circ} \mathrm{C}-195^{\circ} \mathrm{C}$. UV (EtOH) $\lambda_{\max } 303 \mathrm{~nm}$. IR $(\gamma$ $\left.\mathrm{cm}^{-1}\right)$ : 3264 and $1631\left(\mathrm{NH}_{2}\right), 1681(\mathrm{C}=\mathrm{O}), 1601,1507(\mathrm{C}=\mathrm{N}), 1320$ (cyclic NCSN), 1225 (C-F), $1156(\mathrm{C}-\mathrm{S})$, 826 (p-substituted phenyl), 618 (C-F). ${ }^{1} \mathrm{H}$ NMR ( $\left.\mathrm{DCCl}_{3}-\mathrm{d}_{6}\right) \delta$ (ppm): 8.61 - 7.84, 7.84 - 7.83, 7.82 - 7.81, 7.818 7.812, 7.24,7.15,7.14, 7.13, 7.115, 7.110 (aromatic CH), 3.5 (s, $\left.2 \mathrm{H}, \mathrm{NH}_{2}\right) \cdot{ }^{13} \mathrm{C}$ NMR $\left(\mathrm{DCCl}_{3}-\mathrm{d}_{6}\right) \delta(\mathrm{ppm}): 165$, 163, 160, 130.58, 130.49, 130.37, 116.15, 115.93, 77.34, 77.03, 76.71. M/Z: (Int.\%): $357\left(57 \% \mathrm{M}^{+} \mathrm{H}_{2} \mathrm{O}\right) 244$ (1.0), 206 (15.0), 178 (100), 134 (45), 95 (25.0). CHNSF analysis for $\mathrm{C}_{16} \mathrm{H}_{9} \mathrm{~N}_{5} \mathrm{SF}_{2} \mathrm{O}$ (357), Calcd: C, 53.78; $\mathrm{H}$, 2.52; N, 19.60; S, 8.63; F, 10.64\%. Found: C, 53.58; H, 2.32; N, 19.55; S, 8.33; F, 10.43\%.

\subsection{N-(Cyclohexyl)-N'-[4'-oxo-1,2,4-triazino[3,4-b] [1,3,4]thiadiazol-7'- (4"-fluorophenyl)-3'-(4"-fluorophenyl)]-urea (2).}

A mixture of compound $1(0.01 \mathrm{~mol})$ and cyclohexyl isocyanate $(0.01 \mathrm{~mol})$ in DMF (50 ml) warmed for $2 \mathrm{~h}$, cooled then poured onto ice. The solid produced filtered off and crystallized from dioxan to give $\mathbf{2}$ as faint yellow. Yield 60\%, m.p. $178^{\circ} \mathrm{C}-180^{\circ} \mathrm{C}$. IR $(\gamma) \mathrm{cm}^{-1}$ : $3325(\mathrm{NH}), 2928,2851$ (aliphatic $\left.\mathrm{CH}, \mathrm{CH}_{2}\right) 1700(\mathrm{C}=\mathrm{O})$, 1629 (CONH), 1602 (C=N), 1413 (deformation $\mathrm{CH}_{2}$ ), 1320 (cyclic NCSN), 1225 (C-F), 1155 (C-S), 826, 796 (p-substituted phenyl), 635 (C-F). ${ }^{1} \mathrm{H}$ NMR (DMSO-d 6 ) $\delta$ (ppm): 8.73 (s, 2H, NH, NH), 7.97, 7.96, 7.95, 7.94 (4H, aryl protons), 7.38, 7.37, 7.35 (3H, aryl protons), 2.511, 1.8, 1.7, 1.6, 1.2, (each s, $11 \mathrm{H}$, aliphatic protons). ${ }^{13}$ C NMR (DMSO-d ${ }_{6}$ ) $\delta$ (ppm): 168.4, 163.4 (2 C=O), 160 (C-S), 130.71, 130.65, 130.38, 130.36 (aromatic carbons), 116, 116.1 (C-F), 33.32, 25.28, 24.45 (aliphatic carbons). CHNSF analysis for $\mathrm{C}_{23} \mathrm{H}_{20} \mathrm{~N}_{6} \mathrm{SF}_{2} \mathrm{O}_{2}(482$ ). Calcd: C, 57.26; H, 4.14; N, 17.42; S, 6.6; F, 7.88\%. Found: C, 56.96; H, 4.05; N, 17.11; S, 6.33; F, 7.75\%.

\subsection{N-(4'-Fluorophenyl)-N'-[(4'-oxo-1',2',4'-triazino[3,4-b] [1,3,4] thiadiazol-7'- (4"-fluoro-phenyl)-3"-(4"-fluorophenyl)] thiourea (3)}

A mixture of 1 (0.01 mol) and 4-fluorophenyl isothiocyanate $(0.01 \mathrm{~mol})$ in DMF (20 ml) was refluxed for $1 \mathrm{~h}$, cooled then poured onto ice. The yielded solid filtered off and crystallized from EtOH to give $\mathbf{3}$ as yellow crystals. Yield 72\%, m.p. $164^{\circ} \mathrm{C}-165^{\circ} \mathrm{C}$. IR $(\gamma) \mathrm{cm}^{-1}$ : $3272(\mathrm{NH}), 3016$ (aromatic $\left.\mathrm{CH}\right), 1695(\mathrm{C}=\mathrm{O}), 1612(\mathrm{C}=\mathrm{N})$, 1600 (C=C), 1328 (cyclic NCSN), 1225 (C-F), 1208 (C=S), 1154 (C-S), 829,732, 719 (p-substituted phenyl), 639 (C-F). ${ }^{1} \mathrm{H}$ NMR (DMSO-d ${ }_{6}$ ) $\delta$ (ppm): 9.76, 8.73 (each s, 2H, NH), 7.97, 7.95, 7.47, 7.46, 7.45, 7.44, 7.38, 7.35, 7.18, 7.14, 7.11 (m, m, $11 \mathrm{H}$, aromatic protons). ${ }^{13} \mathrm{C}$ NMR (DMSO-d $\left.\mathrm{d}_{6}\right) \delta(\mathrm{ppm}): 180.29(\mathrm{C}=\mathrm{S}), 164.7$ 
(C=O), 135.98, 135.62, 135.61 (C-N), 130.71, 126.24 (aromatic carbons), 119.97, 119.92 (C-N), 116.14, 115.32, 114.98 (C-F). CHNSF analysis for $\mathrm{C}_{23} \mathrm{H}_{13} \mathrm{~N}_{6} \mathrm{~S}_{2} \mathrm{~F}_{3} \mathrm{O}$ (510). Calcd: C, 54.11; H, 2.54; N, 16.47; S, 12.54; F, 11.17\%. Found: C, 53.98; H, 2.48; N, 16.24; S, 12.38; F, 10.27\%.

\subsection{3-[(2'-Acetylamino)-5'-fluorophenyl]-7-(4'-fluorophenyl)-1,2,4-triazino[3,4-b] $[1,3,4]$ thiadiazol-4-one (4)}

A mixture of $1(0.200 \mathrm{gm})$ and glacial acetic acid $(5 \mathrm{ml})$ was warmed for $5 \mathrm{~min}$, cooled then poured onto ice. The solid produced filtered off and crystallized from AcOH to give $\mathbf{4}$ as pall yellow crystals. Yield 55\%, m.p. $182^{\circ} \mathrm{C}-183^{\circ} \mathrm{C}$. IR $(\gamma) \mathrm{cm}^{-1}$ : $3123(\mathrm{NH}), 1632(\mathrm{C}=\mathrm{O}), 1603(\mathrm{C}=\mathrm{N}), 1414$ (deformation $\left.\mathrm{CH}_{3}\right), 1321$ (cyclic NCSN), 1225 (C-F), 1155 (C-S), 827, 795 (p-substituted phenyl), 635 (C-F). ${ }^{1} \mathrm{H}$ NMR (DMSO-d $\left.\mathrm{d}_{6}\right) \delta$ (ppm): $8.73(\mathrm{~s}, 1 \mathrm{H}, \mathrm{NH}), 7.97,7.96,7.95,7.94(\mathrm{~m}, 4 \mathrm{H}$, aromatic protons), 7.38, 7.37, 7.3 (m, 3H, aromatic protons) 2.51 (s, 3H, $\left.\mathrm{CH}_{3}\right) .{ }^{13} \mathrm{C}$ NMR (DMSO-d $\left.{ }_{6}\right) \delta(\mathrm{ppm}): 164.71(\mathrm{C}=\mathrm{O}), 160.50(\mathrm{C}=\mathrm{O}), 130.71,130.65,130.36$, (aromatic carbons), 116.15, 116.0 (C-F), $39.03\left(\mathrm{CH}_{3}\right)$. CHNSF analysis for $\mathrm{C}_{18} \mathrm{H}_{11} \mathrm{~N}_{5} \mathrm{SF}_{2} \mathrm{O}$ (399). Calcd: C, 54.13; H, 2.75; N, 17.54; S, 8.0; F, 9.52\%. Found: C, 53.88; H, 2.69; N, 17.32; S, 7.75; F, 9.40\%.

\subsection{3-(2'-Trifluoroacetylamino-5'-fluorophenyl)-7-(4'-fluorophenyl)-1,2,4-triazino $[3,4-b][1,3,4]$ thiadiazol-4-one (5)}

Equimolar mixture of $\mathbf{1}$ and trifluoroethyl acetate in THF (50 ml) refluxed for $2 \mathrm{~h}$, cooled. The solid thus obtained filtered off and crystallized from dioxan to give 5 as white crystals. Yield $82 \%$, m.p. $179^{\circ} \mathrm{C}-180^{\circ} \mathrm{C}$. IR $(\gamma)$ $\mathrm{cm}^{-1}$ : 1632 (C=O), 1603 (C=N), 1321 (cyclic NCSN), 1225 (C-F), 1155 (C-S), 827, 795 (p-substituted phenyl), 635 (C-F). ${ }^{1} \mathrm{H}$ NMR (DMSO-d $\left.{ }_{6}\right) \delta(\mathrm{ppm}): 8.73(\mathrm{~s}, 1 \mathrm{H}, \mathrm{NH}), 7.97,7.96,7.95,7.94$ (4H, aromatic protons), 7.38, 7.37, 7.35 (3H, aromatic protons). ${ }^{13} \mathrm{C}$ NMR (DMSO-d $\left.\mathrm{d}_{6}\right) \delta(\mathrm{ppm}): 164.71(\mathrm{C}=\mathrm{O}), 160.50(\mathrm{C}=\mathrm{O}), 130.71,130.65$, 130.38, 130.36 (aromatic carbons), 116.14, 116.00 (C-F). M/S (Int.\%): 455 (M+2, 0.11\%), 190 (100), 153 (85.0), 138 (3.33), 95 (100), 66 (1.181, 60, 13.0). CHNSF analysis for $\mathrm{C}_{18} \mathrm{H}_{8} \mathrm{~N}_{5} \mathrm{SF}_{2} \mathrm{O}_{2}$ (455, M+2). Calcd: C, 47.68; H, 1.76; N, 15.45; S, 7.06; F, 20.97\%. Found: C, 47.38; H, 1.73; N, 15.19; S, 6.88; F, 20.67\%.

\subsection{0-(4'-Fluorophenyl)-4-fluoro-1,3,4-thiadiazolo[2,3-c][1,2,4] triazino[5,6-b]indole (6)}

Compounds $1(0.20 \mathrm{gm})$ in DMF $(20 \mathrm{ml})$ refluxed for $3 \mathrm{~h}$, cooled then poured onto ice. The yielded solid filtered off and crystallized from EtOH to give 6 as yellowish crystals. Yield $60 \%$, m.p. $184^{\circ} \mathrm{C}-185^{\circ} \mathrm{C}$. IR $(\gamma) \mathrm{cm}^{-1}$ : 1603 (C=N), 1321 (cyclic NCSN), 1226 (C-F), 1155 (C-S), 827, 796 (p-substituted phenyl), 635 (C-F). ${ }^{1} \mathrm{H}$ NMR (DMSO-d $\left.\mathrm{d}_{6}\right) \delta$ (ppm): 7.97, 7.96, 7.95, 7.94 (4H, aromatic protons), 7.38, 7.37, 7.35 (3H, aromatic protons). ${ }^{13} \mathrm{C}$ NMR (DMSO-d $\left.\mathrm{d}_{6}\right) \delta$ (ppm): 130.71, 130.65, 130.38, 130.36 (aromatic carbons), 116.14, 116.0 (C-F). CHNSF analysis for $\mathrm{C}_{16} \mathrm{H}_{7} \mathrm{~N}_{5} \mathrm{SF}_{2}$ (339). Calcd: C, 56.63; H, 20.6; N, 20.6; S, 9.43; F, 11.20\%. Found: C, 56.55; H, 2.03; N, 20.18; S, 9.14; F, 10.98\%.

\subsection{3-(2'-Diphenylphosphatoamino-5'-fluorophenyl)-7-(4'-fluorophenyl)-1,2,4- triazino[3,4-b][1,3,4]thiadiazol-4-one (7)}

An equimolar mixture of $\mathbf{1}(0.01 \mathrm{~mol})$ and diphenyl phosphoryl chloride $(0.01 \mathrm{~mol})$ in DMF $(20 \mathrm{ml})$ refluxed for $30 \mathrm{~min}$, then, cooled and poured onto ice. The produced solid filtered off and crystallized from THF to give $\mathbf{7}$ as yellowish crystals, yield $60 \%$, m.p. $173^{\circ} \mathrm{C}-175^{\circ} \mathrm{C}$. IR $(\gamma) \mathrm{cm}^{-1}$ : 3061(aromatic $\mathrm{CH}$ ), $1632(\mathrm{C}=\mathrm{O}), 1602(\mathrm{C}=\mathrm{N})$, 1320 (cyclic NCSN), 1225 (C-F), 1155 (C-S), 1097 (P=O), 1016 (Ph-O-P), 962, 936, 870, 827, 795 (substituted phenyl), 635 (C-F). ${ }^{1} \mathrm{H}$ NMR (DMSO-d 6 ) $\delta$ (ppm): 8.73 (s,1H, NH), 7.97, 7.96, 7.95, 7.94 (4H, aromatic protons), 7.38, 7.37, 7.35 (3H, aromatic protons), 7.2-6.8 (m,10H, phenyl protons). ${ }^{13} \mathrm{C}$ NMR (DMSO-d $\left.\mathrm{d}_{6}\right) \delta(\mathrm{ppm})$ : 160.50 (C=O), 130.71, 130.65, 130.38, 130.36 (aromatic carbons), 116.14, 116.0 (C-F). M/S (Int.\%):590 (M+, 0.11\%), 248 (100), 247 (11.8), 95 (95.0). CHNSF analysis for $\mathrm{C}_{28} \mathrm{H}_{18} \mathrm{~N}_{5} \mathrm{SF}_{2} \mathrm{PO}_{4}$ (589). Calcd: C, 57.04; H, 3.05; N, 11.88; S, 5.43; F, 6.45\%. Found: C, 57.01; H, 2.98; N, 11.60; S, 5.37; F, 6.14\%.

\subsection{3-[5'-Fluoro-2'-(4"-fluorobenzoylamino)-phenyl]-7-(4'-fluorophenyl)-1,2,4- triazino $[3,4-\mathrm{b}][1,3,4]$ thiadiazol-4-one $(8)$}

A mixture of $1(0.01 \mathrm{~mol})$ and 4-fluorobenzoyl chloride $(0.01 \mathrm{~mol})$ in DMF $(20 \mathrm{ml})$ refluxed $1 \mathrm{~h}$, cooled. The reaction mixture poured onto ice. The solid produced filtered off and crystallized from dioxan to give $\mathbf{8}$ as yel- 
lowish crystals, yield $65 \%$, m.p. $149^{\circ} \mathrm{C}-150^{\circ} \mathrm{C}$. IR $(\gamma) \mathrm{cm}^{-1}$ : 3200-3100 (b, OH, NH), 1677 (cyclic C=O), 1633 (NHCO), 1602 (C=N), 1315 (cyclic NCSN), 1225 (C-F), 1156 (C-S), 827, 796, 768 (p-substituted phenyl), 635 (C-F). ${ }^{1} \mathrm{H}$ NMR (DMSO-d $\left.{ }_{6}\right) \delta$ (ppm): $13.09(\mathrm{~s}, 1 \mathrm{H}, \mathrm{NH}), 8.02,8.05,8.01,(\mathrm{~m}, 3 \mathrm{H}$, aromatic protons), 7.97, 7.96, 7.95, 7.94 (m, 4H, aromatic protons), 7.38-7.32 (m, 4H, aromatic protons). ${ }^{13} \mathrm{C}$ NMR (DMSO- $\left.\mathrm{d}_{6}\right) \delta$ (ppm): 166.36, 164.04 (2 C=O), 132.11, 132.05, 130.65, 130.38, 130.36, 127.33 (aromatic carbons), 116.15, 116.0 (C-F), 115.69, 115.54 (C-N). CHNSF analysis for $\mathrm{C}_{22} \mathrm{H}_{12} \mathrm{~N}_{5} \mathrm{SF}_{3} \mathrm{O}_{2}$ (467). Calcd: C, 56.53; H, 2.56; N, 14.98; $\mathrm{S}$, 6.85; F, 12.20\%. Found: C, 56.28; H, 2.50; N, 14.53; S, 6.67; F, 12.00\%.

\subsection{3-(5'-Fluoro-2'-carboxymethylanilino)-7-(4'-fluorophenyl)-1,2,4-triazino[3,4-b] $[1,3,4]$ thiadiazol-4-one (9)}

A mixture of $\mathbf{1}(0.01 \mathrm{~mol})$ and monochloroacetic acid $(0.01 \mathrm{~mol})$ in DMF $(20 \mathrm{ml})$ refluxed for $1 \mathrm{~h}$, cooled then poured onto ice. The solid produced filtered off and crystallized from THF to give $\mathbf{9}$ as yellowish crystals, yield $72 \%$, m.p. $180^{\circ} \mathrm{C}-182^{\circ} \mathrm{C}$. IR $(\gamma) \mathrm{cm}^{-1}$ : 3500 - 3100 (b, OH, NH), 1720, 1633 (2 C=O), 1603 (C=N), 1490, 1413 (deformation $\mathrm{CH}_{2}$ ), 1321 (cyclic NCSN), 1226 (C-F), 1156 (C-S), 826, 796 (substituted phenyl), 636 (C-F). ${ }^{1} \mathrm{H}$ NMR (DMSO-d $\left.\mathrm{d}_{6}\right) \delta(\mathrm{ppm}): 8.73(\mathrm{~s}, 1 \mathrm{H}, \mathrm{NH}), 7.97,7.96,7.95,7.94(\mathrm{~m}, 4 \mathrm{H}$, aromatic), 7.38, 7.37, $7.35(\mathrm{~m}, 3 \mathrm{H}$, aromatic), 4.55 (s, 1H, OH), 2.51 (s, 2H, J,8.7, $\left.\mathrm{CH}_{2}\right) .{ }^{13} \mathrm{C}$ NMR (DMSO-d $\left.\mathrm{d}_{6}\right) \delta(\mathrm{ppm}): 164.71(\mathrm{C}=\mathrm{O}), 163.06$ $(\mathrm{C}=\mathrm{O}), 130.70,130.65,130.38,130.36$ (aromatic carbons), 116.14, 116.0 (C-F), $39.03\left(\mathrm{CH}_{2}\right)$. CHNSF analysis for $\mathrm{C}_{18} \mathrm{H}_{11} \mathrm{~N}_{5} \mathrm{SFO}_{3}(415)$. Calcd: C, 52.53; H, 2.65; N, 16.86; S, 7.71; F, 9.15\%. Found: C, 52.33; H, 2.62; N, 16.59; S, 7.62; F, 9.04\%.

\subsection{3-(5'-Fluoro-2'-carboxymethinicanilino)-7-(4'-fluorophenyl)-1,2,4-triazino $[3,4-b][1,3,4]$ thiadiazol-4-one (10)}

A mixture of $1(0.01 \mathrm{~mol})$ and 1,1'-dichloroacetic acid $(0.01 \mathrm{~mol})$ in DMF $(20 \mathrm{ml})$ refluxed for $30 \mathrm{~min}$, cooled then poured onto ice. The solid thus obtained filtered off and crystallized from EtOH to give $\mathbf{1 0}$ as yellowish crystals, yield 60\%, m.p. 154-155 ${ }^{\circ}$. IR $(\gamma) \mathrm{cm}^{-1}$ : $3500-3300(\mathrm{OH}), 1696,1632(\mathrm{C}=\mathrm{O}), 1602(\mathrm{C}=\mathrm{N}), 1321$ (cyclic NCSN), 1226 (C-F), 1155 (C-S), 826, 796 (substituted phenyl), 635 (C-F). ${ }^{1} \mathrm{H}$ NMR (DMSO-d 6 ) $\delta$ (ppm): $8.8(\mathrm{~s}, 1 \mathrm{H}, \mathrm{N}=\mathrm{CH}), 7.97,7.96,7.95,7.94(\mathrm{~m}, 4 \mathrm{H}$, aromatic), 7.38, 7.37, $7.35(\mathrm{~m}, 3 \mathrm{H}$, aromatic), $4.41(\mathrm{~s}, 1 \mathrm{H}, \mathrm{OH})$. ${ }^{13} \mathrm{C}$ NMR (DMSO-d $\left.\mathrm{d}_{6}\right) \delta(\mathrm{ppm}): 164.71(\mathrm{C}=\mathrm{O}), 163.06(\mathrm{C}=\mathrm{O}), 130.71,130.65,130.38,130.36,128.72,127.25$ (aromatic carbons), 116.14, 116.0 (C-F). CHNSF analysis for $\mathrm{C}_{18} \mathrm{H}_{9} \mathrm{~N}_{5} \mathrm{SFO}_{3}(413)$. Calcd: C, 52.30; H, 2.17; N, 16.94; S, 7.74; F, 9.20\%. Found: C, 52.08; H, 2.11; N, 16.55; S, 7.54; F, 8.89\%.

\subsection{3-(5"-Fluoro-2'-cyanomethylanilino)-7-(4'-fluorophenyl)-1,2,4-triazino[3,4-b] $[1,3,4]$ thiadiazol-4-one (11)}

Equimolar amounts of $\mathbf{1}$ and chloroacetonitrile in DMF (20 ml) refluxed for $1 \mathrm{~h}$, cooled then poured onto ice. The solid produced filtered off and crystallized from THF to give $\mathbf{1 1}$ as brown crystals, yield $65 \%$, m.p. $177^{\circ} \mathrm{C}$ $178^{\circ} \mathrm{C}$. IR $(\gamma) \mathrm{cm}^{-1}$ : $3180(\mathrm{NH}), 2220(\mathrm{C} \mathrm{N}), 1635(\mathrm{C}=\mathrm{O}), 1507,1413$ (deformation $\mathrm{CH}_{2}$ ), 1320 (Cyclic NCSN), 1225 (C-F), 1155 (C-S), 226, 796 (Substituted phenyl), 635 (C-F). ${ }^{1} \mathrm{H}$ NMR (DMSO-d $\left.{ }_{6}\right) \delta$ (ppm): 8.73 (s,1H, $\mathrm{NH}), 7.97,7.96,7.95,7.94$ (4H, aromatic), 7.38, 7.37, 7.35 (m, 3H, aromatic), 3.36 (2H, J, 6.6 p.c.s, $\left.\mathrm{CH}_{2}\right) .{ }^{13} \mathrm{C}$ NMR (DMSO- $\left.\mathrm{d}_{6}\right) \delta$ (ppm): $160.50(\mathrm{C}=\mathrm{O}), 130.71,130.65,130.38,130.36,128.91,127.88$ (aromatic carbons), 116.14, 116.0 (C-F), $39.03\left(\mathrm{CH}_{2} \mathrm{CN}\right)$. CHNSF analysis for $\mathrm{C}_{18} \mathrm{H}_{10} \mathrm{~N}_{6} \mathrm{SF}_{2} \mathrm{O}(396)$. Calcd: C, 54.54; H, 2.52; N, 21.21; S, 8.08; F, 9.59\%. Found: C, 54.31; H, 2.50; N, 20.89; S, 7.98; F, 9.41\%.

\subsection{3-(5'-fluoro-2'-Methylanilino)-7-(4'-Fluorophenyl)-1,2,4-Triazino[3,4-b][1,3,4] Thiadi-Azol-4-One (12)}

A mixture of $9(0.20 \mathrm{gm})$ and $\mathrm{K}_{2} \mathrm{CO}_{3}$ solution ( $\left.5 \%, 50 \mathrm{ml}\right)$ refluxed for $1 \mathrm{~h}$, cooled then acidification use $5 \% \mathrm{HCl}$. The solid obtained filtered off and crystallized from EtOH to give $\mathbf{1 2}$ as brownish crystals, yield 55\%, m.p. $178^{\circ} \mathrm{C}-180^{\circ} \mathrm{C}$. IR $(\gamma) \mathrm{cm}^{-1}$ : 3062 (aromatic CH), 1700, $1632(\mathrm{C}=\mathrm{O}), 1600(\mathrm{C}=\mathrm{N}), 1507,1413$ (deformation $\mathrm{CH}_{3}$ ), 1321 (cyclic NCSN), 1225 (C-F), 1155 (C-S), 820, 795 (Substituted phenyl), 635 (C-F). ${ }^{1} \mathrm{H}$ NMR (DMSO-d $\left.\mathrm{d}_{6}\right) \delta(\mathrm{ppm}): 8.73(\mathrm{~s}, 1 \mathrm{H}, \mathrm{NH}), 7.97,7.96,7.95,7.94(\mathrm{~m}, 4 \mathrm{H}$, aromatic), 7.38, 7.37, 7.35 (m, 3H, aromatic), $2.22\left(\mathrm{~s}, 3 \mathrm{H}, \mathrm{CH}_{3} \mathrm{~N}\right) .{ }^{13} \mathrm{C}$ NMR (DMSO-d $\left.\mathrm{d}_{6}\right) \delta(\mathrm{ppm}): 160.11(\mathrm{C}=\mathrm{O}), 130.71,130.65,130.38,130.36$, 128.72 (aromatic carbons), 116.14, $116.0(\mathrm{C}-\mathrm{F}), 22.65\left(\mathrm{CH}_{3}\right)$. CHNSF analysis for $\mathrm{C}_{17} \mathrm{H}_{11} \mathrm{~N}_{5} \mathrm{SF}_{2} \mathrm{O}(371)$. Calcd: C, 
54.98; H, 2.96; N, 18.86; S, 8.62; F, 10.24\%. Found: C, 54.69; H, 2.22; N, 18.43; S, 8.49; F, 9.98\%.

\subsection{Formation of 9}

A mixture of $\mathbf{1 1}(0.20 \mathrm{gm})$ and diluted $\mathrm{HCl}(10 \%, 50 \mathrm{ml})$ refluxed for $1 \mathrm{~h}$, coled. The solid produced filtered off and crystallized from THF to give 9 as yellowish crystals, yield $60 \%$, m.p. $178^{\circ} \mathrm{C}-179^{\circ} \mathrm{C}$. Mixed melting point no depression.

\subsection{N'[2'-(4"-Fluorophenyl)-5-oxo-6-(5'-fluorophenyl-2"-yl)-N3-(4'-fluorophenyl)- thiobarbituric acid (13)}

Equimolar mixture of $\mathbf{3}$ and malonic acid in glacial acetic acid $(20 \mathrm{ml})$ refluxed for $4 \mathrm{~h}$, cooled and poured onto ice. Extracted the organic layer by diethyl ether and leaf at room temperature. The solid obtained crystallized from dioxan to give 13 faint yellow crystals, yield $65 \%$, m.p. $150^{\circ} \mathrm{C}-151^{\circ} \mathrm{C}$. IR $(\gamma) \mathrm{cm}^{-1}: 3530(\mathrm{OH}), 1660$ (C=O), 1488 (deformation $\mathrm{CH}_{2}$ ), 1385 (NCSN), 1255 (C-F), 1205 (C=S), 670 (C-F). ${ }^{1} \mathrm{H}$ NMR (DMSO-d 6 ) $\delta$ (ppm): $10.04(\mathrm{~s}, 1 \mathrm{H}, \mathrm{OH}), 8.2$ - 8.0 (m, 4H, aromatic protons), 7.60-7.44 (m, 4H, aromatic protons),7.0 - 6.98 (m, $3 \mathrm{H}$, aromatic protons), 3.55, $2.59-2.58$ (s, $\left.2 \mathrm{H}, \mathrm{CH}_{2}\right) .{ }^{13} \mathrm{C}$ NMR (DMSO- $\mathrm{d}_{6}$ ) $\delta$ (ppm): 181.10, 165.71, 160.00, 159.38, 138.2, 133.40, 134.20, 126.91, 126.80, 125.1, 121.69, 115.45, 115.21, 77.79, 77.57, 77.36, 44.36, 40.46-39.8). CHNSF analysis for $\mathrm{C}_{36} \mathrm{H}_{13} \mathrm{~N}_{6} \mathrm{~S}_{2} \mathrm{~F}_{3} \mathrm{O}_{3}$ (578). Calcd: C, 53.97; H, 2.24; N, 14.53; S, 11.67; F, 9.86\%. Found: C, 53.79; H, 2.11; N, 14.33; S, 11.55; F, 9.59\%.

\section{Pharmacological Evaluation}

1,2,4-Triazine derivatives showed a wide biocidal spectrum [13]-[15]. Also, 1,3,4-thiadiazoles exhibited a large biocidal agents [11] [12]. In addition, introduction of both fluorine atoms and/or amino groups to heterocyclic systems often improve their medicinal properties [5]-[9]. Thus, in search for new drugs as potential anthelmintic to control on the smoke diseases, the present work, aim to obtain new drugs. All the new synthesized compounds were screened for their anthelmintic activity against $\mathrm{H}$. nana infection in mice, by using the standard method of steward. The oral dose was $200 \mathrm{mg} / \mathrm{Kg}$ given for 2 days. Only the compounds 1, 3, 5, 6, 7, 9 and 13 recorded a weak activity $(10<\%)$. On the other hand, evaluation of these compounds against N-brasiliensis infection in rats ta the same oral dose, by using other standard method [26] [27]. The obtained results showed that the activity in range of $25 \%-60 \%$ (Table 1 ).

From the obtained results (Table 1) we can be conclude that compounds containing $\mathrm{COCF}_{3}$ are highly effect than aromatic C-F. also, presence of phosphate group bonded to NH enhance that activity. Full fluorinated

Table 1. Anthelmintic activity of the new synthesized compounds.

\begin{tabular}{cccc}
\hline Compound No. & H. nana & N. brasiliensis \\
$\mathbf{1}$ & $10<$ & 55 \\
$\mathbf{2}$ & $10<$ & 29 \\
$\mathbf{3}$ & $10<$ & 35 \\
$\mathbf{4}$ & $10<$ & 60 \\
$\mathbf{5}$ & $10<$ & 45 \\
$\mathbf{6}$ & $10<$ & 58 \\
$\mathbf{7}$ & $10<$ & 32 \\
$\mathbf{8}$ & $10<$ & 48 \\
$\mathbf{9}$ & $10<$ & 29 \\
$\mathbf{1 0}$ & $10<$ & 30 \\
$\mathbf{1 1}$ & $10<$ & 30 \\
$\mathbf{1 2}$ & $10<$ & 49 \\
\hline
\end{tabular}


$\mathrm{N}, \mathrm{N}$-diarylthiourea showed a rise activity towards $\mathrm{N}$-brasifiensis. As well as $\mathrm{N}$-alkyl systems exhibited a moderate activity. Thus, atype of both compounds $\mathbf{5}$ and $\mathbf{7}$ would present a fruitful matrix for the future development of a new class of potential anthelmintic agents, that deserves further investigation and derivation. A simple of nucleophilic attack of amino group of fluorinated 1,2,4-triazino[2,3-c]thiadiazolone to various electrophilic agents was deduced to give $\mathrm{N}$-substituted analogues. The anthelmintic activity of these systems was evaluated. Among these tested analogs, compounds 5 and $\mathbf{7}$ showed 50\% - 60\% activity, while all the tested compounds exhibited below $10 \%$ activity towards H. nana.

\section{References}

[1] Al-Harbi, A.S., Abdel-Rahman, R.M. and Asiri, A.M. (2014) Synthesis of Some New Fluorine Substituted Thiobarbituric Acid Derivatives as Anti-HIV1 and Cyclin-Dependent Kinase 2(CDK $)$ for Cell Tumor Division-Part II. International Journal of Organic Chemistry, 4, 142-153. http://dx.doi.org/10.4236/ijoc.2014.42016

[2] Makki, M.S.T., Abdel-Rahman, R.M. and Khan K.A. (2014) Fluorine Substituted 1,2,4-Triazinones as Potential Anti-HIV-1 and CDK $_{2}$ Inhicitors. Journal of Chemistry, 2014, Article ID: 430573.

[3] Makki, M.S.T., Abdel-Rahman, R.M., Faidallah, H.M. and Khan K.A. (2013) Synthesis of New Fluorine Substituted Heterocyclic Nitrogen Systems Derived from p-Aminosalicyclic Acid as Anti-Mycobacterial Agents. Journal of Chemistry, 2013, Article ID: 819462.

[4] Makki, M.S.T., Abdel-Rahman, R.M., Faidallah, H.M. and Khan, K.A. (2013) Synthesis of Substituted Thioureas and Their Sulfur Heterocyclic Systems of p-Aminosalicylic Acid as Anti-Mycobacterial Agents. Journal of Chemistry, 2013, Article ID: 862463.

[5] Abdel-Rahman, R.M. and Ali, T.S. (2013) Synthesis and Biological Evaluation of Some New Polyfluorinated 4-Thiazolidinone and $\alpha$-Aminophosphonic Acid Derivatives. Monatshefte für Chemie, 144, 1243-1252. http://dx.doi.org/10.1007/s00706-013-0934-6

[6] Makki, M.S.T., Bakhotmah, D.A., Abdel-Rahman, R.M. and El-shahawy, M.S. (2012) Designing and Synthesis of New Fluorine Substituted Pyrimidine-Thion-5-Carbonitriels and the Related Derivatives as Photochemical Probe Agents for Inhibition of Vitiligo Diseases. International Journal of Organic Chemistry, 2, 311-320.

[7] Makki, M.S.T., Bakhotmah, D.A. and Abdel-Rahman, R.M. (2012) Highly Efficient Synthesis of Novel Fluorine Bearing Quinolone-4-carboxylic Acid the Related Compounds as Amylolytic Agents. International Journal of Organic Chemistry, 2, 49-55. http://dx.doi.org/10.4236/ijoc.2012.21009

[8] Abdel-Rahman, R.M., Makki, M.S.T. and Bawazir, W.A. (2011) Synthesis of Some More Fluorine Heterocyclic Nitrogen Systems Derived from Sulfa Drugs as Photochemical Probe Agents for Inhibition of Vitiligo Disease-Part I. E-Journal of Chemistry, 8, 405-414. http://dx.doi.org/10.1155/2011/586063

[9] Abdel-Rahman, R.M., Makki, M.S.T. and Bawazir, W.A. (2010) Synthesis of Some More Fluorine Heterocyclic Nitrogen Systems Derived from Sulfa Drugs as Photochemical Probe Agents for Inhibition of Vitiligo Disease-Part II. E-Journal of Chemistry, 7, S93-S102.

[10] Abdel-Rahman, R.M. (2001) Chemistry of Uncondensed 1,2,4-Triazines, Part IV—Synthesis and Chemistry of Bioactive 3-Amino-1,2,4-triazines and Related Compounds. Pharmacies, 56, 275-286.

[11] Shawali, A.S, (2014) 1,3,4-Thiadiazoles of Pharmalogical Interest: Resent Trends in Their Synthesis via Tandem 1,3Dipolar-Cycloaddition: Review. Journal of Advanced Research, 5, 1-17. http://dx.doi.org/10.1016/j.jare.2013.01.004

[12] Kushwaha, N., Kushwaha, S.K.S. and Rai, A.K. (2011) Biological Activities of Thiadiazole Derivatives. International Journal of ChemTech Research, 4, 517-531.

[13] Abdel-Rahman, R.M., Makki, M.S.T., Ali, T.S. and Ibrahim, M.A. (2012) 1,2,4-Triazine Chemistry Part III-Synthesis Strategies to Functionalized Brideghead Nitrogen Hetero Annulated 1,2,4-Triazine Systems and Their Region Specific and Pharmacological Properties. Current Organic Synthesis, 9, 1-25.

[14] Abdel-Rahman, R.M., Makki, M.S.T., Ali, T.S. and Ibrahim, M.A. (2010) 1,2,4-Triazine Chemistry Part I: Orientation of Cyclization Reaction of Functionalized 1,2,4-Triazine Derivatives. European Journal of Chemistr, 1, $236-245$. http://dx.doi.org/10.5155/eurjchem.1.3.236-245.54

[15] Abdel-Rahman, R.M., Makki, M.S.T., Ali, T.S. and Ibrahim, M.A. (2014) 1,2,4-Triazine Chemistry Part IV: Synthesis and Chemical Behavior of 3-Functionalized-5,6-Diphenyl-1,2,4-Triazines towards Some Nucleophilic and Electrophilic Reagents. Journal of Heterocyclic Chemistry, ID JHET, 12-0734. http://dx.doi.org/10.1002/jhet.2014

[16] El-Gendy, Z., Morsy, J.M., Allimony, H.A., Abdel-Monem, W.R. and Abdel-Rahman, R.M. (2001) Synthesis of Heterobicyclic Nitrogen Systems Bearing the 1,2,4-Triazine Moiety as Anti-HIV and Anticancer Drugs, Part III. Pharmazie, 56, 376-383.

[17] Makki, M.S.T., Abdel-Rahman, R.M. and Abu-Ali, O.A.(2015) Synthesis of Some More New Fluorinated 1,2,4-Tri- 
azino[3,4-b][1,3,4] Thiadiazolones and Their Molluscicidal Against Selective Snails-Part I. Journal of Chemistry and Chemical Engineering, 9, 162-175.

[18] Abdel-Rahman, R.M. (1991) Synthesis and Anti-Human Immune Virus Activity of Some New Fluorine Containing Substituted 3-Thioxo-1,2,4-Triazin-5-Ones. Farmaco, 46, 379-389.

[19] Abdel-Rahman, R.M. (1992) Synthesis of New Fluorine Bearing Trisubstituted 3-Thioxo-1,2,4-Triazin-5-Ons as Potential Anticancer Agents. Farmaco, 47, 319-326.

[20] Al-Romazian, A.N., Makki, M.S.T. and Abdel-Rahman, R.M. (2014) Synthesis of New Fluorine/Phosphorus Substituted 6-(2'-Aminophenyl)-3-Thioxo-1,2,4-5(2H,4H)One and Their Related Alkylated Systems as Molluscicidal Agent as against the Snails Responsible for Bilharziiasis Diseases. International Journal of Organic Chemistry, 4, 154-168. http://dx.doi.org/10.4236/ijoc.2014.42017

[21] Breuer, E. (1996) The Chemistry of Organophosphorus Compounds. John Wiley and Sons, NewYork.

[22] Blakley, B., Broussea, U., Fournier, M. and Voccia, A.I. (1999) Immunotoxicity of Pesticides: A Review. Toxicology and Industral Health, 15, 119-132. http://dx.doi.org/10.1177/074823379901500110

[23] Ibrahim, M.A., Abdel-Rahman, R.M., Abdel-Halim, A.M., Ibracim, S.S. and Allimony, H.A., (2008) Synthesis and Antifungal Activity of Novel Polyheterocyclic Compounds Containing 1,2,4-Triazine Moiety. Archive for Organic Chemistry, 2008, 202-215. http://dx.doi.org/10.3998/ark.5550190.0009.g19

[24] Abdel-Rahman, R.M. and Islam, E.I. (1993) Synthesis and Reactions of Acetonitrile Dervatives Bearing a 5,6-Diphenyl-1,2,4-Tryazin-3-yl Moiety. Indian Journal of Chemistry, 32, 526-529.

[25] Al-Harbi, A.S., Abdel-rahman, R.M. and Asiri, A.M. (2015) Synthesis of Some New Fluorine Substituted Thiobarbituric Acid Derivatives as Anti HIV-1 and Cyclin-Dependent Kinase 2(CDK2) for Cell Tumor Division-Part I. European Journal of Chemistry, 6, 63-70. http://dx.doi.org/10.5155/eurjchem.6.1.63-70.1147

[26] Steward, J.S. (1955) Anthelmintic Studies: II. A Double Entero-Nemacidal Anthelmintic Test Covering a Wide Range of Activities. Parasitology, 45, 242-254. http://Dx.Doi.Org/10.1017/S003118200002761x

[27] Stadden, O.P. (1963) Experimental Chemotherapy. Academic Press, New York. 